\title{
Remote observing with the Keck Telescope from California using NASA's ACTS satellite
}

Patrick L. Shopbell, Judith G. Cohen, Larry A. Bergman

Patrick L. Shopbell, Judith G. Cohen, Larry A. Bergman, "Remote observing with the Keck Telescope from California using NASA's ACTS satellite," Proc. SPIE 3112, Telescope Control Systems II, (18 September 1997); doi: 10.1117/12.278835

SPIE Event: Optical Science, Engineering and Instrumentation '97, 1997, San Diego, CA, United States 


\title{
Remote observing with the Keck Telescope from California using NASA's ACTS satellite
}

\author{
P. L. Shopbell ${ }^{a}$, J. G. Cohen ${ }^{a}$, and L. Bergman ${ }^{b}$ \\ ${ }^{a}$ California Institute of Technology, Mail Stop 105-24, Pasadena, CA 91125 USA \\ ${ }^{b}$ Jet Propulsion Laboratory, Mail Stop 525-3660, Pasadena, CA 91109 USA
}

\begin{abstract}
As a technical demonstration project for the NASA Advanced Communications Technology Satellite (ACTS), we have implemented remote observing on the 10-meter Keck II telescope on Mauna Kea in Hawaii from the California Institute of Technology campus in Pasadena. The data connection consists of ATM networks in Hawaii and California, running at OC-1 speeds (51 Mbit/sec) through optical fiber, and high data rate (HDR) satellite antennae at JPL in Pasadena and at the Tripler Army Medical Center in Honolulu. The ACTS network provides sufficient bandwidth to enable true remote observing, with a software environment identical to that used for on-site observing.

In this paper, we demonstrate that while the satellite link introduces a number of difficulties and decreases overall reliability of the system, remote observing is not only feasible, but provides several important advantages over standard observing paradigms. Benefits include involving more members of observing teams while decreasing expenses, enhancing real-time data analysis of observations by persons not subject to altitude-related conditions, and providing facilities, expertise, and personnel not normally available at the observing site. Although the current bandwidth of the public Internet is insufficient for true remote observing, we nevertheless anticipate a growing role for remote observing techniques, particularly as high-speed terrestrial networking paradigms, such as ATM, become more commonly available.
\end{abstract}

Keywords: remote observing, Keck Observatory, ACTS, satellite communications, ATM

\section{INTRODUCTION}

Remote use of astronomical telescopes has been a topic of interest for many years, since space-based observing platforms (e.g., IUE) began to demonstrate total remote operation out of sheer necessity. Initially, a number of ground-based radio and optical telescopes (e.g., the WIYN Telescope ${ }^{1}$ ) introduced queue-based scheduling, a mixture of remote and interactive observing modes. Only very recently are optical telescopes beginning to realize the benefits of true remote observing: for example, observations with modest size detectors at Apache Point Observatory are being carried out remotely using the Internet. ${ }^{2}$ In this project, we have established remote interactive observing capabilities for Keck Observatory (http: //www2.keck. hawaii.edu:3636/) on Mauna Kea for observers at Caltech, in Pasadena, California. In undertaking this project, we were motivated by several operational and scientific advantages that remote observing would offer.

One primary concern is the high altitude of the Keck Observatory. At 13,600 feet of elevation, the summit of Mauna Kea is a demanding location for both mental and physical exertion. In spite of the requirement that all astronomers spend a night at Hale Pohaku (altitude $\approx 9000$ feet) for acclimatization before proceeding to the summit for a night of observing, about $15 \%$ of the people who do not observe often at Mauna Kea become sufficiently ill during the course of a 3 night run that they have to leave the summit for at least 12 hours. Approximately $75 \%$ of the people coming to the summit to observe for a full night experience some discomfort such as a mild headache, and almost all experience some loss of judgment, irritability, etc. Remote observing provides an environment for all observers that is free of these difficulties, and also provides an opportunity for people who cannot tolerate high altitudes (e.g., pregnant women, those with heart conditions, etc.) to observe with the Keck Telescopes.

Further author information:

P.L.S. (correspondence): Email: pls@astro.caltech.edu; WWW: http://astro.caltech.edu/ ppls

J.G.C.: Email: jlc@astro.caltech.edu

L.B.: Email: larry@jplopto.jpl.nasa.gov 
Another logistic motivation for remote observing involves issues common to large telescopes located at sites distant from the home institutions: In general, the larger the telescope, the more heavily over-subscribed it is. Runs are therefore often only 1 or 2 nights in duration. Since 2-3 observers come to each run, this means substantial sums of money are spent on travel and related expenses. The additional night of acclimatization for high-altitude sites such as Mauna Kea increases the cost further. Finally, the salary cost for "wasted time" during these runs is quite large. An excellent example of the potential savings is that of the European Southern Observatory (ESO; http://www.hq.eso.org/): There are 19 telescopes near the Atacama desert in Chile, including two 3.6-meter telescopes and a set of four 8-meter giants currently under construction (the Very Large Telescope, or VLT). The observing site is widely regarding as one of the very best in the world, yet it is half-way around the globe from most of its large European user population. Understandably, remote observing is gaining popularity among European astronomers. $^{3}$

Remote diagnosis of hardware and software problems also becomes more feasible with an operational remote observing system. In our case, the teams that built the instrument hardware and software for the Keck Telescopes are located at Caltech or a campus of the University of California. Just their presence in the same buildings as the remote observers can be extremely helpful when problems arise in the operation of the telescope. The establishment of remote observing from California implies the presence of a network connection, which can allow engineers and programmers to analyze the remote systems essentially instantaneously. Again, both travel and time are saved, and effective help from highly skilled and experienced people in California can be obtained quickly when necessary.

In addition to these operational advantages, there are strong scientific advantages to remote observing as well. With remote observing, every member of a large collaboration can participate in obtaining the data. It is possible for one part of the team to concentrate on obtaining the observations, while other team members can be analyzing the scientific results from the last observation, checking the instrumental performance to make sure everything is working correctly (particularly the detector), or browsing the literature or catalogs of objects as necessary to prepare for the next set of observations. The inclusion of students in the observing session becomes much easier, cheaper, and more routine when no travel is required, i.e., they don't need to miss classes. The facilities available at remote observing sites (e.g., Caltech) usually far exceed those available at the observatory site, whether it be computer hardware, office and library supplies, or a pizza delivery. Recall also that the remote site may be located such that the night hours of observing overlap, or even coincide with standard business hours at the remote site.

Given these strong motivations for remote observing, there are then several issues which must be examined in order to make remote observing feasible, from both technical and operational viewpoints. The primary issue involves the large size of astronomical images. The optical instruments in use at the Keck Telescope have frames that are currently 8 Megabytes in size, soon to become a factor of 4 larger. Although actual integration times depend on the scientific program, and range from less than a second to an hour, the quality of ground based optical and infrared astronomy observations is very sensitive to weather conditions, including clouds and atmospheric turbulence. Hence, even though observing sessions are planned in detail in advance, careful "quick look" analysis of each image is important in defining what to do next, how long the next exposure should be, whether to switch to brighter objects due to poor sky conditions, how to modify the program to cope with unexpected failures of non-critical telescope or instrument components, etc. An operating mode such as this clearly requires a means of viewing or retrieving the images at the remote observing site with a minimal amount of delay. The public Internet connection between Hawaii and California was in 1995 (and still is today) insufficient for these purposes.

We therefore submitted a proposal to NASA as part of its Advanced Communications Technology Satellite (ACTS) Gigabit Satellite Network (GSN) testbed program. We received funding to establish a high-speed ATM network running from the dome of the 10-meter Keck Telescopes on the summit of Mauna Kea in Hawaii to the Caltech campus in Pasadena, California, using the ACTS satellite as the network link across the Pacific Ocean. This network has been used to support remote observing, remote diagnosis of problems, remote software development, and other related tasks. In the sections that follow, we will outline the network architecture and topology, describe the role of the ACTS satellite in our system, demonstrate the capabilities and remote operation of a specific instrument on the Keck II Telescope, and summarize the benefits and difficulties which we have encountered during the course of this ACTS demonstration project. 


\section{NETWORK ARCHITECTURE}

We now describe the topology of our remote observing network, the primary protocols involved, and our experience with high-speed satellite networking for a remote observing application.

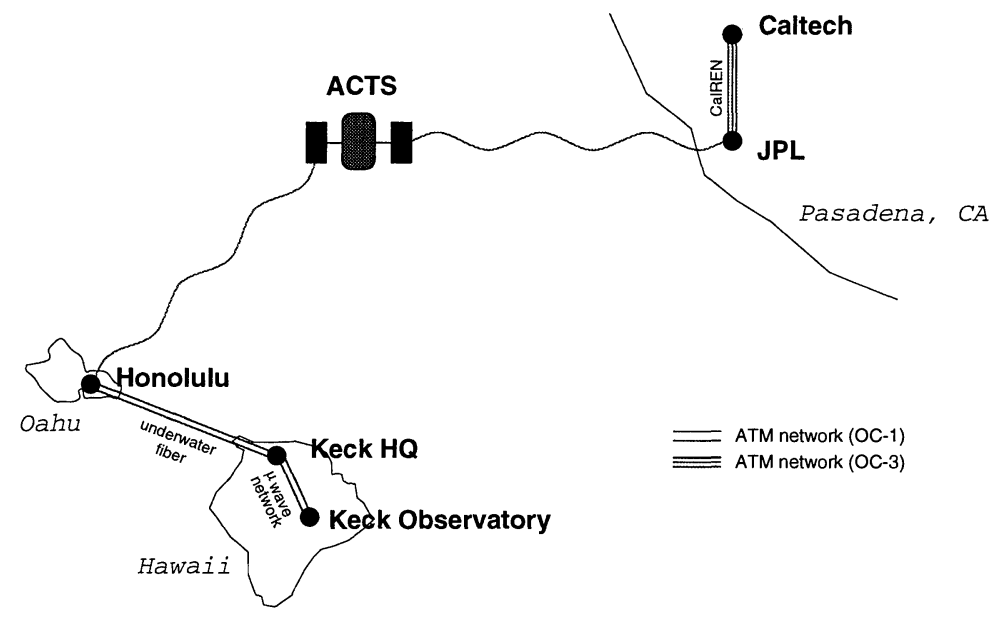

Figure 1. A schematic of the high-speed ACTS network used for the Keck remote observing project. Note that the entire ground-based network now consists of optical fiber.

\subsection{Physical layer}

When first established in 1996, our network consisted of several different physical media, including optical fiber, satellite stations, and microwave antennae, as illustrated in Figure 1. The network consists of three major segments: the ground network in California, the satellite link across the Pacific Ocean, and the ground network in Hawaii.

The ground network in California connects Caltech with JPL, the site of the satellite ground station. This portion of the network was established as part of Pacific Bell's extant fiber optic network. The only work required to establish this physical connection was the installation of a fiber optic line from the Caltech backbone to the remote observing room. This portion of the network has also been the most static, remaining essentially the same for the past year of operation.

The ground network in Hawaii has been somewhat more complex in its evolution, primarily due to the relative inexperience of GTE Hawaiian Telephone, as compared to PacBell in California, and a lack of infrastructure in Hawaii. Initially, the network consisted of undersea optical fiber for a portion of its length, from the satellite ground station in Honolulu to the GTE Hawaiian Telephone office on the big island of Hawaii. (This fiber had been installed less than a year before our project began.) The network then utilized microwave antennae to reach Hale Pohaku, at the 9,000-foot level on Mauna Kea, as fiber optical cable had not yet been installed in these remote areas. The final portion of the network, from Hale Pohaku to the telescope dome on the summit of the mountain, again employs fiber optic cable.

In January of 1997, the portion of the ground network in Hawaii consisting of microwave antennae was finally upgraded to optical fiber (see Figure 2). The improved performance for high-speed data transfers resulting from the lower bit error rates (BER) of the final all-fiber network was immediately noticed.

\subsection{Network protocol layer}

The lowest-level protocol used by this network is the standard Synchronous Optical Network (SONET) optical data transmission protocol. This is the level at which the ACTS satellite operates, i.e., it knows nothing of protocols above the raw SONET data stream.

In order to enable standard higher-level networking protocols, we installed an Asynchronous Transfer Mode (ATM) network as our base data protocol. ATM is a packet-switched protocol, similar to frame relay, which is 


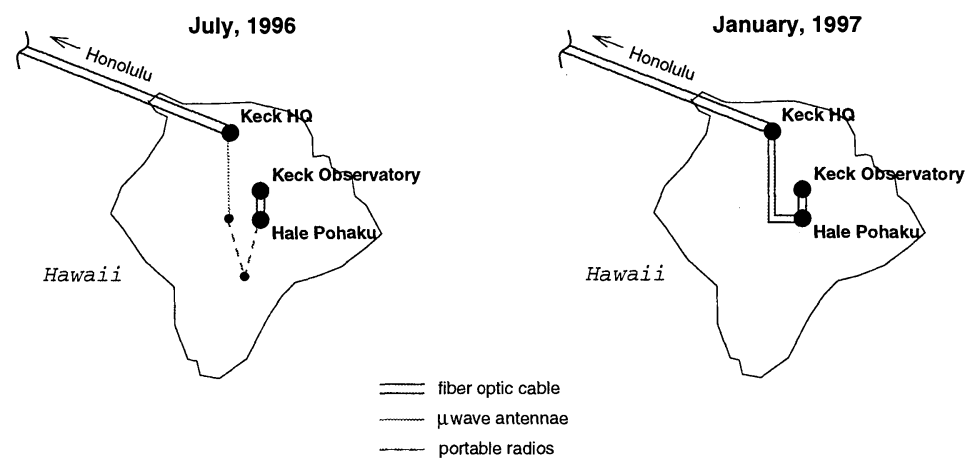

Figure 2. A schematic of the high-speed terrestrial network in Hawaii used for the Keck remote observing project. The network is shown in its initial and final (all-fiber) states.

capable of bandwidths exceeding $2 \mathrm{Gbit} / \mathrm{sec}^{4,5}$ Data is transferred in 53 -byte "cells", each containing a 5 byte header and 48 bytes of payload. The transfer of cells is performed by hardware switches, which have been installed throughout the network in California and Hawaii. The ground network in California includes three ATM switches: one at each endpoint (Caltech and JPL), and an intermediate switch belonging to the CalREN (California Research and Education Network) project of Pacific Bell. The ground network in Hawaii includes several ATM switches: one at each endpoint (Honolulu and the Keck Telescope dome), and a number of intermediate switches belonging to GTE Hawaiian Telephone.

The ATM switches provide a point-to-point network connecting the computer in the remote observing room at Caltech with the instrument control computer at the Keck Telescope in Hawaii. We have established Permanent Virtual Circuits (PVCs) in each of the switches, which direct the cells between the endpoints of the network. Several vendors have supplied the ATM switches for this network, including FORE Systems, Newbridge Networks, and SynOptics. Fore ATM Network Interface Cards (NICs) are used to interface the Sun SPARCStation 20/51 workstations at each end of the network. This mixed vendor environment has been a stringent test of the compatibility among vendors in the relatively new ATM environment. Although we have encountered several problems associated with interoperability issues, none have been extremely serious, and the ATM vendors and telephone companies have been extremely helpful in attempting to diagnose and solve ATM-level problems. We have also witnessed the increasing popularity of ATM even in the mere 2-year lifetime of this project, and expect to see more widespread use of this protocol at the WAN and enterprise network levels. (For more information on ATM, see http://cell-relay.indiana.edu/cell-relay/.)

\subsection{User protocol layer}

ATM is not a "reliable" protocol in the networking sense - cells may be dropped or lost in transit, and no attempt is made to verify delivery. In order to facilitate reliable data transfer, and to allow the use of the wealth of software tools already available, we are running the standard IP protocols over ATM. We are using a pseudo-standard implementation known as "Classical IP", which defines a relationship between standard IP "dot" addressing and ATM PVCs, as well as data packet segmentation and reassembly algorithms for converting between IP packets and ATM cells. ${ }^{6}$ Since the ATM switches know nothing of the protocol levels above ATM, the choice of this protocol affects merely the two endpoint systems. Those workstations both employ FORE SBA-200 ATM interface cards which perform IP packet segmentation and reassembly in hardware, enabling net ATM speeds of $155 \mathrm{Mbit} / \mathrm{sec}$ (OC-3).

Over the Classical IP level, we run the same TCP/IP and UDP/IP protocols that are used over standard Ethernet* networks. Tools such as ftp, telnet, and the X Window System are part of every observing run, as are additional tools, such as an audio conferencing tool (rat) and a shared whiteboard application (wb).

The most important impact of a satellite component on a high-speed network is the relatively large delay introduced by the round-trip signal travel time to the satellite. In our network, this travel time is approximately 0.55

\footnotetext{
${ }^{*}$ Ethernet is a registered trademark of Xerox Corporation.
} 
seconds, which corresponds to over 3 Mbytes of data at OC-1 speeds (51 Mbit/sec). The problem has to do with the connection-oriented nature of TCP/IP: TCP sends a very specific amount of data, known as a "window", after which time it expects an acknowledgement from the other end of the connection. This is the manner in which TCP/IP is able to implement a "reliable" connection. However, this window size is often very small; the default value for workstations running the SunOS 4.1.4 operating system is 4 Kbytes. If one were to use this system on our satellite network in its default configuration, a window of data would be sent in 0.0007 seconds, after which the sending system would be forced to wait 0.549 seconds for an acknowledgement. In other words, the system would be running at $0.1 \%$ efficiency, and the net throughput would reflect this: initial tests of our system under such conditions showed bandwidths of $0.1-0.2 \mathrm{Mbit} / \mathrm{sec}$.

Fortunately, this problem is well-known in the high-speed networking community. Networks such as ours are known as "long fat networks" (LFN). The figure of merit for such networks is the window size, or the bandwidthdelay product:

$$
\text { TCP window size }=\text { bandwidth } * \text { delay time }
$$

There is an Internet RFC (Request For Comment) document on this subject, RFC $1323,{ }^{7}$ and the problem has been discussed extensively. Many current operating systems support the RFC 1323 extensions, and provide options to increase the TCP window size to values in excess of 10 Mbytes. In the case of the SunOS operating system (to which we are constrained by legacy control software at Keck), we obtained the TCP-LFN package from Sun Consulting, which also purports to support the RFC 1323 extensions for long fat networks. In reality, a number of limitations of SunOS conspire to prohibit one from obtaining extremely large window sizes. In our case, the kernel limit of 2 Mbytes of Mbuf memory (IP packet wrappers) turned out to be the major constraint, limiting our window size to no more than 1 Mbyte. This is approximately one-third of the optimal value derived above, and, as such, we obtain maximum TCP/IP throughputs of approximately 10-15 Mbit/sec, about one-third of the $51 \mathrm{Mbit} / \mathrm{sec}$ OC-1 network operating speed. Although perhaps disappointing in a relative sense, this bandwidth is far in excess of T1 Ethernet speed $(1.44 \mathrm{Mbit} / \mathrm{sec})$ and allows an $8 \mathrm{Mbyte}$ image to be transferred in approximately 5 seconds (not including protocol overhead). As a further comparison, this bandwidth exceeds that which is available on the local area Ethernet network at the Keck Telescope itself.

\section{THE ACTS CONNECTION}

The main deterrent to the implementation of remote observing has always been the problem of obtaining an affordable and reliable connection with adequate bandwidth. NASA's Advanced Communications Technology Satellite was built as a prototype system to explore new modes of high speed transmission for digital data. It provides this capability at rates reaching up to $\mathrm{OC}-12(622 \mathrm{Mbit} / \mathrm{sec})$ via advanced on-board switching and multiple dynamically hopping spot beam antennas for selected areas of the United States, including Pasadena and Hawaii, although the steerable antenna used to reach sites not in the continental U.S. is only capable of OC-3 (155 Mbit/sec) speed. The $20-30 \mathrm{GHz}$ frequency band, not previously used by a communication satellite, is utilized, with extensive rain fade compensation.

ACTS was launched on September 12, 1993 by Space Shuttle Discovery and now occupies a geostationary orbit at $100^{\circ}$ west longitude. It has survived almost twice as long as its planned mission duration of two years, but is now nearing the end of its lifetime, which is limited by the fuel resources required to maintain its stationary position. (Current plans involving steerable ground stations may be implemented to extend the usable lifetime of the satellite even further.) BBN designed, built, and maintains the high data rate (HDR) ground stations that provide a gateway between ACTS and ground-based fiber optic networks and supercomputer interfaces. Five of the semiportable HDR terminals have been built; they are allocated to the various ACTS experiments for pre-determined lengths of time, then moved to another location. (For more information on the ACTS satellite and program, see http://mrpink.lerc.nasa.gov/gsnhome.html.)

Each HDR ground station includes a 12 -foot dish permanently pointed at the satellite and an equipment trailer containing a real-time Unix control system with SONET I/O boards, burst modem, and high-output transmitter. Due to the experimental nature of these ground stations, the often harsh environmental conditions, and the inherent complexity of high-speed communications equipment, the HDR stations have proved to be the weakest link in our network. We have been forced to await replacement of two transmitters, the operating system, and several other HDR components, due to hardware failures. Although the ACTS personnel and BBN have been extremely cooperative in 
restoring service on such occasions, the impact of the reliability issue is that at least one observer must be sent to Hawaii to use the telescope, in case of ACTS-related equipment malfunctions.

The remote nature of most high-quality observing sites exacerbates this problem. Even in Hawaii, BBN maintains only a small field office, making HDR maintenance costly and time-consuming. A truly remote site, which would most benefit from remote observing techniques, also requires the highest degree of robustness from the equipment. In our opinion, the ACTS system is insufficiently robust to provide true remote observing with large (i.e., highly competitive) telescopes, due primarily to its limited scope and experimental nature. However, one of the ACTS Project's primary goals is to stimulate commercial high-speed communications satellite development. These systems may eventually play a role in remote astronomical observing systems.

Another difficulty we have encountered is that the transmitters in the ACTS HDR stations are not designed to run continuously, due to the finite lifetime of certain critical components, but rather must be switched on and off as needed. This method of operation demands human intervention at the beginning and end of every satellite session, a procedure that has been non-trivial to organize and would prove difficult in more remote observatory locations. The Hawaii location itself poses a final problem for us due to the large yearly rainfall at the location of the HDR in Honolulu. Because the frequency band at which ACTS operates is highly susceptible to rain fade, we have lost several runs in the past year due to rain in Honolulu. In essence, the use of the ACTS system for remote observing adds a weather constraint such that it must be clear (i.e., not raining) at both of the ground station sites, as well as at the observatory itself! (We note that this is another area in which future commercial high-speed communications satellites may provide improvements over ACTS.)

\section{KECK OBSERVATORY AND THE LRIS INSTRUMENT}

The W. M. Keck Observatory, located on the summit of Mauna Kea on the Big Island of Hawaii, consists of twin 10-meter telescopes intended for astronomical observations at optical and infrared wavelengths. Both telescopes employ a revolutionary design, in which each 10-meter mirror consists of 36 2-meter hexagonal segments which are aligned to act as a single large mirror. These are the largest astronomical telescopes in the world for use at these wavelengths. The first Keck Telescope has been in routine operation since 1993, and has successfully demonstrated the viability of the multiple-segment mirror design. The second Keck Telescope became operational in October of 1996 and is currently dedicated almost entirely to optical observations.

Throughout the design process of the hardware and software for the Keck Telescopes, the possibility of implementing remote observing, particularly from Waimea, the location of the Keck headquarters in Hawaii, was kept in mind. The instruments, their motors, and the detectors are operated through workstations that are located in the control room of the Keck Telescope dome. It is not necessary during normal night-time operation to go out to the instrument on the telescope to make any adjustments or changes.

We have concerned ourselves exclusively with enabling remote observing on the Keck II telescope with the Low Resolution Imaging Spectrograph ${ }^{8}$ (LRIS). This is the primary optical instrument at the observatory, and the only instrument capable of obtaining direct images at optical wavelengths. It is used on Keck II almost every night of the year. Although our efforts have concentrated on remote observing with LRIS, we note that all of the instrument interfaces have been engineered in a similar fashion, so our results could be easily extended to other instruments on Keck I or II.

Figure 3 illustrates the organization of the telescope and instrument control hardware at the Keck Observatory. The majority of the complications associated with remote control of the instrument have stemmed from security issues and the desire to not impact normal (i.e., non-remote) observations with the telescope. For example, concerns about the effect of a such a high-speed network, especially in various failure modes, on other computer and network systems at Keck, as we approached this project with our initial inexperience, forced us to isolate remote operations more thoroughly by duplicating the instrument control computer. This machine provides boot information for the instrument motor and CCD detector VME crates, as well as the interface between the user and the control systems. The remote portion of the observing system, the workstation at Caltech, is integrated into the system merely as a remote display, using the X Window System protocols. Although this is perhaps not the most efficient method of providing remote operations, it is certainly the most straightforward, especially in a relatively new and frequently changing facility such as Keck. 


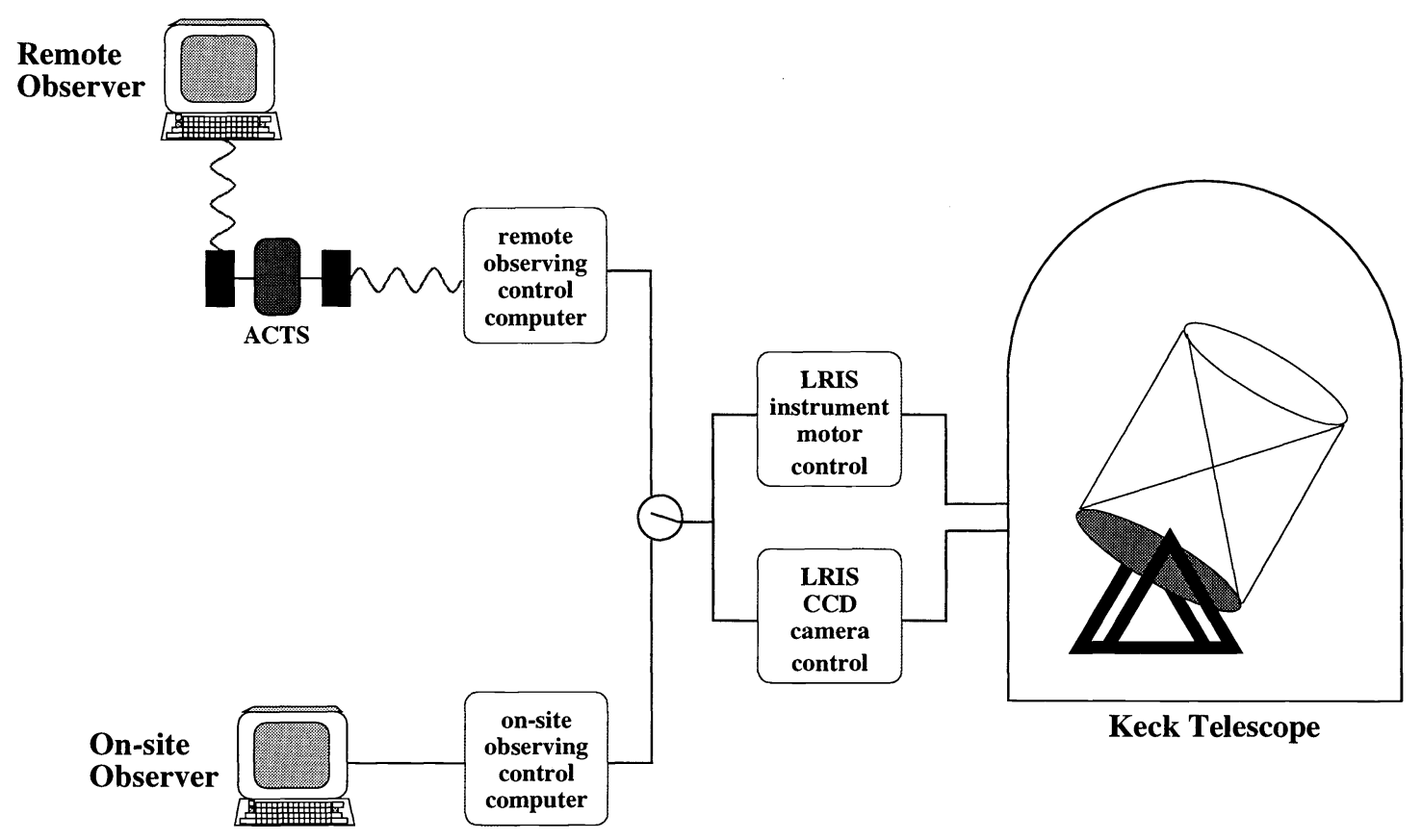

Figure 3. A schematic of the LRIS instrument control system at Keck Observatory. Note that there are two separate instrument control computers: one for standard on-site observations, and another for remote observations over the high-speed network.

We also note that remote observing at Keck now routinely takes place from the observatory headquarters in Waimea, at the base of Mauna Kea. ${ }^{9}$ Although this capability does not reduce travel costs or time (except perhaps eliminating the single day of altitude acclimatization), it does facilitate engineering efforts from Keck Headquarters and eliminates altitude-related problems. For this purpose, the Keck Observatory has installed a PictureTel videoconferencing system for full interaction between the telescope observing assistant and the remote astronomers. The instrument control and operation is implemented in the same way as for our remote satellite-based system: remote display of windows using X Window System protocols.

\section{REMOTE OBSERVING OPERATION}

We now outline a night from a typical remote observing run at Caltech, to demonstrate the advantages and problems associated with such operation.

ACTS scheduling For the testbed ACTS experiments such as ours, the satellite must be scheduled 1-2 weeks ahead of time. This is not a problem in most cases, since observing schedules at Keck are established 6 months at a time. The primary difficulty in scheduling ACTS sessions lies in the 5-hour time difference (6 hours during daylight savings time) between Hawaii (HST) and the East coast (EST). Since the satellite experiences higher demand during daylight hours, it is often difficult to run an observing session remotely during the second half of the Hawaiian night. We therefore often restrict remote runs to the first half of the night. But even this is not a critical problem, as the University of California actually allocates its Keck time in half nights, to provide more astronomers with an opportunity to use the telescopes. And in many full-night runs, the first half of an observing night is the most complex and demanding, so eavesdropping and collaborative use of the remote capabilities are also very useful in that capacity.

ACTS setup When the time comes for the ACTS session to begin, operators at JPL and at the Tripler Army Medical Center in Honolulu will turn on the transmitters at the HDR ground stations. We are fortunate that both HDR units are located at facilities which are in continuous 24-hour operation every day, so that staff 


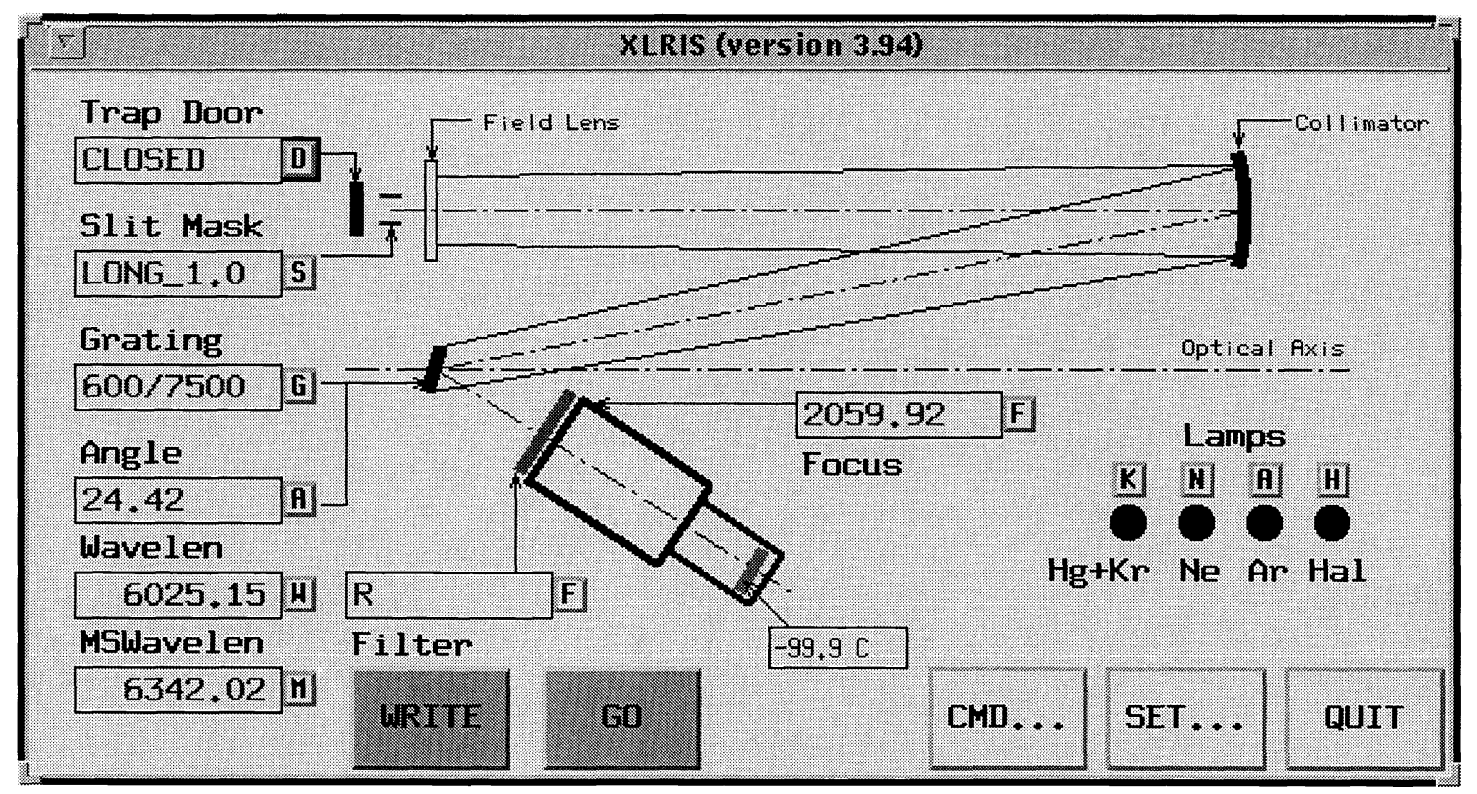

Figure 4. The LRIS instrument control interface at Keck Observatory. This window provides a schematic of the instrument and indicates its current configuration.

are available to turn on/off the ground station transmitters. (As mentioned previously, this arrangement has been arrived at through some negotiation for our experiment, but could prove more difficult for very remote observatory locations.) The ACTS control personnel at NASA/Lewis in Cleveland then connect the satellite with each HDR, and verify that the signal strength is sufficient. Barring hardware complications and rain at either HDR location, the network is generally available within a few minutes of the scheduled time.

Contact Keck Once the network has been established, the observer customarily contacts the observing assistant $(\mathrm{OA})$ at the telescope to indicate that they are ready, to check the weather at the telescope, etc. Also at this stage, an audio link is established over the ATM network, in order to alleviate the need to manipulate (and pay for!) an all-night phone call between the astronomers and the OA. For these purposes, we use a TCP-based audio tool called rat. Based on an earlier tool (vat), rat was developed for the Mbone (Multicast Backbone), but contains full support for point-to-point audio connections. Any of the Internet telephony products commonly available can be used for this purpose. Many include useful features such as echo suppression and voice-activated microphones.

LRIS hardware setup In order to use the LRIS instrument remotely, control must be transferred from the normal instrument control computer at Keck to the duplicate machine which is connected to the ATM network. This involves a 5-minute procedure during which the VME crates which directly control the instrument are instructed to use the alternate machine, and are then rebooted. As described in the previous section, in principle this step is unnecessary, but in practice, security concerns, processing load distribution issues, high-speed networking complications, and the desire to not affect standard Keck observing techniques has led us to establish a separate remote observing control computer. In the future, this rather awkward step should disappear from the remote observing procedure.

LRIS software setup While the OA initializes the telescope control systems, the observer should then start the LRIS instrument control environment. Just as when observing on-site, a single command/menu option starts up all of the necessary tools, with the display optionally redirected to a remote machine. The observer can then verify any requested configuration changes, such as special slitmasks or filters, and set up personalized instrument configuration files. 


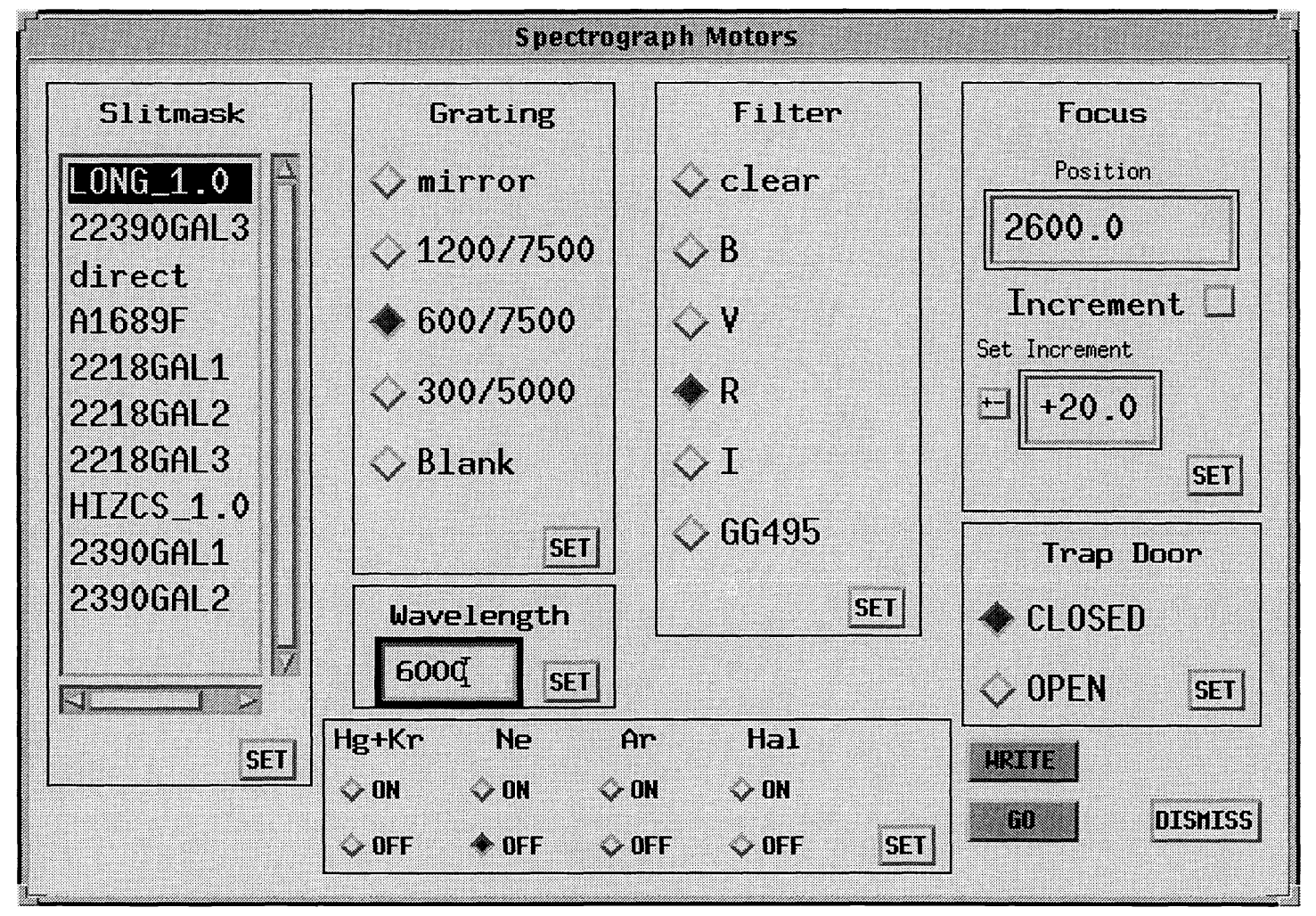

Figure 5. The LRIS instrument control interface at Keck Observatory. This window allows the user to modify the configuration of the instrument.

LRIS operation When both the OA and observer are prepared, the observing session runs in the normal fashion: telescope moves and guiding are handled by the $\mathrm{OA}$, while the observer controls the instrument configuration and CCD exposures. The LRIS instrument is controlled via a graphical interface which provides a schematic of the instrument and its current configuration, along with standard graphical elements (e.g., buttons, lists, etc.) for changing the configuration ${ }^{10}$ (Figures 4 and 5). The CCD camera is controlled through a simple interface which allows the user to set and monitor exposure times (Figure 6). Details such as the number of output CCD amplifiers and the image save directory can be specified as well, of course. A real-time image display is provided, based on the FIGDISP software from FIGARO (Figure 7). Should questions arise, documentation for LRIS and its software are available to the observer via the World Wide Web.

Should any errors be indicated by either the instrument or detector control systems, the OA and/or engineer can be called upon to examine the problem, as with on-site observing. In such situations, we often use a collaborative whiteboard tool, wb, which allows text and graphics to be transmitted in real-time to both parties. This is useful for indicating error messages, describing image characteristics, etc. (As an aside, we note that wb and the rest of the software used for this project is available for free, with the exception of the TCP-LFN software from Sun Consulting.) Should problems arise with the network, personnel may be contacted at the ACTS control center and/or the HDR sites.

Finally, in addition to the instrument control software, all of the usual observing software tools are available remotely: telescope pointing and UT meters, guider window eavesdropping images, etc. Of course, standard TCP tools such as telnet and ftp are used regularly to retrieve images to the local system, where any of several data reduction packages commonly used in astronomical image processing are available. As mentioned above, one of the outstanding features of remote observing is the wealth of familiar software and hardware facilities that are available at the user's home institution: printers, personal workstations and software, libraries, etc. 


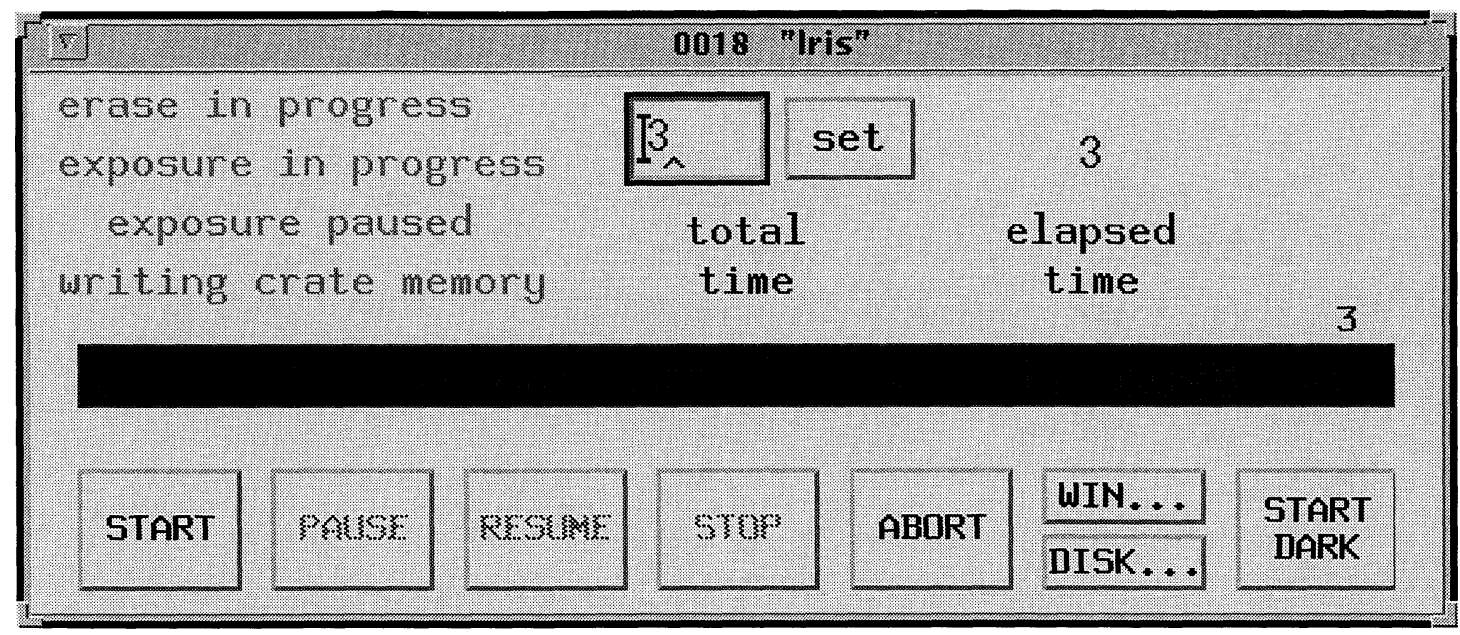

Figure 6. The LRIS instrument control interface at Keck Observatory. This window controls exposures with the CCD camera.

System shutdown Following the end of the observing session, control of the LRIS instrument is returned to the primary computer. This leaves the instrument ready for the next day's engineering and non-remote observing. Finally, a phone call is usually made to the ACTS control center to verify that the run is completed.

\section{CONCLUSIONS}

The Keck/ACTS remote observing project has allowed us to experiment with remote observing on the Keck Telescope with a bandwidth not yet available to Hawaii via terrestrial networks. We have compared a variety of paradigms and tools for remote operations, and evaluated the performance of local tools over a large-bandwidth, long-delay network connection. Our work has led to a number of conclusions, many of which are applicable to ground-based remote observing efforts and non-astronomical communications satellite experiments:

1. Remote observing techniques have the potential to save appreciable expenditures in terms of money and time, while simultaneously enabling increased levels of collaboration in the observing process. In the case of an observatory with large numbers of observers, short observing runs, and/or a very remote site, these savings may very well outweigh initial network costs to enable remote observing.

2. The portable design of the Keck Telescope and instrument control systems has enabled remote observing to be implemented with only relatively minor software modifications. However, additional tools are needed over those available on-site to create a collaborative environment among the remote observing astronomers and the on-site telescope staff. Such tools are becoming widely available with the expansion and increasing popularity of the Internet.

3. At the current time, high-speed terrestrial networks are the most viable source for adequate bandwidth to enable true remote observing. While the ACTS system is not sufficiently robust to enable remote observing, this testbed project suggests that future commercial-grade communications satellites may provide the reliability and affordability necessary for high-bandwidth remote software applications.

4. The most outstanding problem regarding the viability of geosynchronous communications satellites for Internetbased software applications concerns the performance of the standard TCP/IP protocol over high-bandwidth, long-delay time networks. Although the initial set of extensions (i.e., RFC 1323) provide some relief, and several groups (e.g., Mitre Corporation) are working on this problem, its solution may determine the ultimate role for satellite communications in the WAN market. 

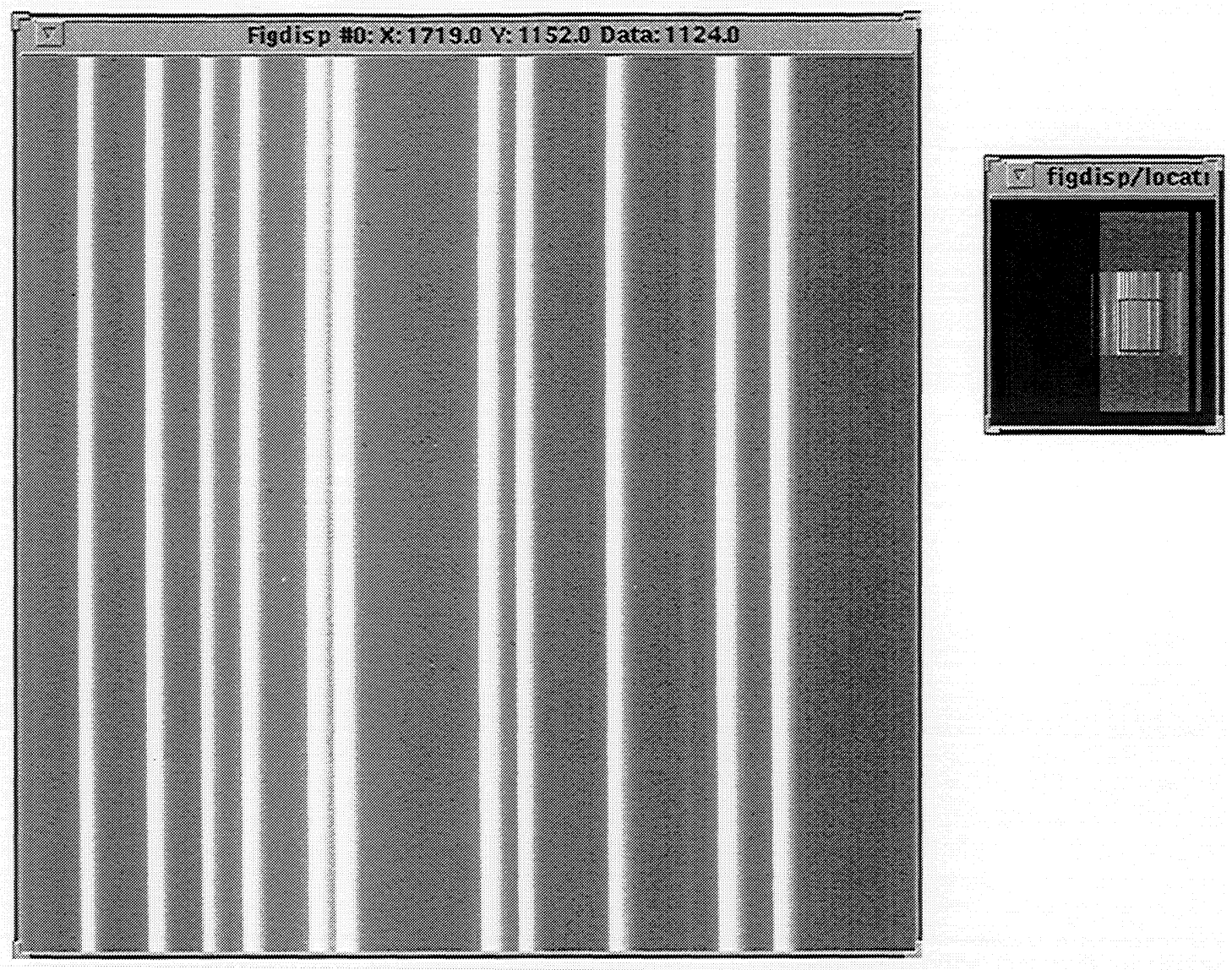

Figure 7. The LRIS instrument control interface at Keck Observatory. This window provides a real-time graphical interface for the observations. Images are transferred directly to this display as the CCD chip is read out.

\section{ACKNOWLEDGEMENTS}

The research described in this paper was performed primarily at the California Institute of Technology and at the Center for Space Microelectronics Technology at the Jet Propulsion Laboratory. It has been sponsored by the National Aeronautics and Space Administration, Office of Advanced Concepts and Technology, under grant BK-50920-42-00-00. The W. M. Keck Observatory is operated as a scientific partnership between the California Institute of Technology and the University of California. It was made possible by the generous gift of the W. M. Keck Foundation, and the support of its late president, Howard Keck. We also wish to thank Jimi Patel and Carl McFadden, our ground station operators at JPL and Tripler Army Medical Center, respectively, and Jon Chock, the Keck Observatory Systems Administrator, for their crucial assistance for the entire duration of this project. The scheduling and operations staff for ACTS have been extremely accommodating as well.

\section{REFERENCES}

1. J. W. Percival, "Remote observing from the bottom up: the architecture of the WIYN telescope control system," in Telescope Control Systems, P. T. Wallace, ed., Proc. SPIE 2479, pp. 33-40, 1995.

2. D. G. York, "The 3.5 meter at Apache Point Observatory," Bull. Am. Ast. Soc. 186, p. 44.04, 1995.

3. A. Wallander, "Operating telescopes remotely," Nuclear Instruments and Methods in Physics, Section A 352, pp. $258-261,1994$.

4. N. K. Cheung, "The infrastructure for gigabit computer networks," IEEE Communications Magazine, pp. 60-68, April 1992. 
5. R. J. Vetter and D. H. C. Du, "Issues and challenges in ATM networks," Communications of the ACM 38, pp. 28-109, 1995.

6. J. D. Cavanaugh and T. J. Salo, "Internetworking with ATM WANs," December 14, 1992.

7. V. Jacobson, R. Braden, and D. Borman, RFC 1323: TCP Extensions for High Performance, May, 1992.

8. J. B. Oke, J. G. Cohen, M. Carr, J. Cromer, A. Dingizian, F. H. Harris, S. Labrecque, R. Lucinio, W. Schaal, H. Epps, and J. Miller, "The Keck Low Resolution Imaging Spectrometer," Pub. Ast. Soc. Pac. 107, p. 375, 1995.

9. A. Conrad, J. Gathright, and R. I. Kibrick, "Remote observing with the Keck Telescopes," in Telescope Control Systems II, H. Lewis, ed., Proc. SPIE 3112, 1997.

10. J. G. Cohen, J. L. Cromer, S. Southard Jr., and D. Clowe, "The software for the LRIS on the Keck 10meter telescope," in Astronomical Data Analysis Software and Systems III, D. R. Crabtree, R. J. Hanisch, and J. Barnes, eds., PASP Conference Series 61, p. 469, 1994. 